\title{
Research on hub location and routing distribution for Hub-and-Spoke logistics network
}

\author{
Yunhe Ma', a , Xifu Wang ${ }^{2, b}$, Jijun Pang ${ }^{3, c}$ \\ ${ }^{1}$ School of Yunhe Ma, Beijing Jiaotong University, Beijing 100044, China; \\ ${ }^{2}$ School of Xifu Wang, Beijing Jiaotong University, Beijing 100044, China; \\ ${ }^{3}$ School of Jijun Pang, Beijing Jiaotong University, Beijing 100044, China; \\ a15120858@bjtu.edu.cn, bxwang1@bjtu.edu.cn, ${ }^{\mathrm{c}} 12120898 @$ bjtu.edu.cn,
}

Keywords: Hub-and-Spoke logistics network, hub location, routing distribution, tabu search.

\begin{abstract}
With the development of the economic globalization, Hub-and-Spoke logistics network has become the effective network structure which could help to collaborate logistics resource, reduce logistics cost and improve operational efficiencies. Therefore, research on location and routing distribution for Hub-and-Spoke logistics network is particularly important. This paper focused on hub location and routing distribution for Hub-and-Spoke logistics network. According to the theory of queuing theory, the hub location is optimized and the design of flow distribution under deliver time requirements is investigated which based on minimizing the total cost in the networks. By reducing the waste of resources in the process of the shipments transportation, the operation efficiency of logistics network is improved and network operators and customers to achieve a win-win objective.
\end{abstract}

\section{Introduction}

In 1986, O'Kelly used the hub logistics network on freight hub location problem the first time, and then put forward for continuous cargo terminal and the discrete type single-allocation hub location models, pointed out that the problem is an NP-hard problem [1]. Campbell made a number of distribution site, for the first time in origin between the paths as decision variables defined in a way, for the entire transport network total minimum cost mixed integer programming model and consider the question of capacity and route development costs [2]. Julia constructing transport networks using hybrid genetic algorithm to solve it, the literature does not take into account emission in direct shipping between the nodes [3].

Firstly, the paper basing on summarizing the literatures of the domestic and abroad, combined the analysis of the developing process and current research status of Hub-and-Spoke logistics network, the bi-level programming model is developed. Using the tabu search to solve the problem. Finally, through the actual data of 6 places, the validity of model and algorithm are verified with MATLAB software.

\section{The Hub-and-Spoke Logistics Network}

The Hub-and-Spoke Logistics Network is a kind of logistics network structures consists of one or more hub as the main shaft, the other more Spoke and the connecting shafts, to achieve transfer of persons, services and goods. As shown in the figure 1 . 


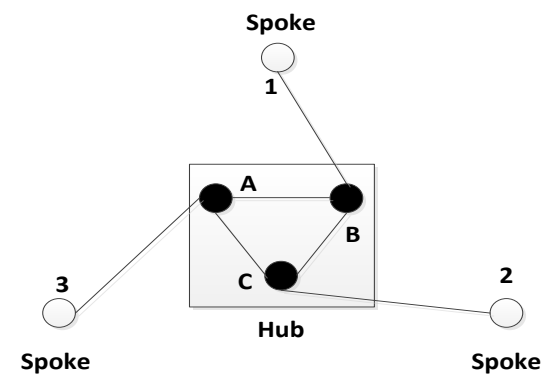

Fig. 1 the structure of the Hub-and-Spoke logistics network

\section{The model}

In the premise of meeting the transportation time limit, select the hub and path allocation scheme, the hub-and-spoke logistics network total cost, design a bi-level programming model. Which the upper model is hub location and path allocation, the lower level is in solving cargo terminal wait time of queuing theory model.

\subsection{The upper model}

The upper model assumes that the following: (1) Road transport is the mode of transportation between radiation node, also for the radiation node and the hub, Railway or road transport is the mode of transportation between the hubs. (2) Assuming that a city can only have one hub, the research only for road and rail transport (3) Time of different modes of transport and the city services time limit are known. You need to consider the transit time of goods and the wait time caused by hub congestion. (4) Transshipment of the goods only in the hub, and the goods transfer twice at most. (5) Terminal fixed operating costs and provide connection of different modes of transport costs are known. (6) The transport costs of goods between cities in the hub-and-spoke logistics network are known. (7) The fixed costs of cities operations hub-and-spoke logistics network are known. (8) According to goods of source and went can will arrived hub of goods is divided into three species: first the Hub Outbound Shipment; second the Hub Inbound Shipment; third the Local Shipment.

Parameters are defined as follows:

$R_{O}$ — - the outbound goods handling process (unloading, delivery, loading);

$R_{I}$ —— the process of the into the station goods (unloading, sorting, loading);

$R_{L}$ — the handling process of the goods in hubs (unloading, sorting, loading);

$\alpha$ - the road transportation discount coefficient, $0<\alpha<1$;

$\mathrm{p}$ - - the number of the hubs;

$f_{i j}$ - the freight from city i to city j;

$c_{i j}^{t}$ - - the unit cost of transport mode $\mathrm{t}$ from city $\mathrm{i}$ to city $\mathrm{j}, \mathrm{t} \in\{1,2\}, \mathrm{t}=1$ the road transport, $\mathrm{t}=2$ the railway transport;

$F_{k}$ - - the fixed costs of city k operations hub-and-spoke logistics network;

$M C_{k t}$ — - the docking fees of hub $\mathrm{k}$ with transport mode $\mathrm{t}$;

$T_{i j}^{t}$ - - the time of transport from city i to city j;

$T W_{i j}$ - - the city services time limit;

$L^{t}$ — - the volume of goods need to be assembled;

$W_{k}^{O}(X)$ - - the average service time when the goods handled by process o in hub k;

The decision variables:

$y_{k}=\left\{\begin{array}{cc}1, & \text { the city } \mathrm{k} \text { is a hub } \\ 0, & \text { otherwise }\end{array}\right.$ 
$X_{i j}^{1}=\left\{\begin{array}{lc}1, & \text { the goods transportation from city } \mathrm{i} \text { to city } \mathrm{j} \\ 0, & \text { otherwiae }\end{array}\right.$

$X_{i j k m}^{t}=\left\{\begin{array}{lc}1, & \text { the goods from city i than city } \mathrm{k} \text { and } \mathrm{m} \text { to city } \mathrm{j} \text { by transport mode } \mathrm{t} \\ 0, & \text { otherwise }\end{array}\right.$

$S_{k t}=\left\{\begin{array}{lc}1, & \text { hub k provides transport mode } \mathrm{t} \\ 0, & \text { otherwise }\end{array}\right.$

The objective function is to minimize the total cost $\mathrm{Z}$ of the network. Total costs fixed by the hub network construction cost, Hub provides the fixed connection between different modes of transport costs and transportation costs of the goods constitutes.

Subject to:

$$
\begin{array}{r}
\text { Min Z }=\sum_{k \in N} F_{k} y_{k}+\sum_{k \in N, t \in T} M C_{k t} S_{k t}+\sum_{i, j, k, m \in N} \alpha c_{k m}^{1} f_{i j} X_{i j k m}^{1}+\sum_{i, j \in N} c_{i j}^{1} f_{i j} X_{i j}^{1} \\
+\sum_{i, j, k, m \in N, t \in T \backslash\{1\}} c_{k m}^{t} \frac{f_{i j}}{L^{t}} X_{i j k m}^{t}+\sum_{i, j, k, m \in N, t \in T} f_{i j}\left(c_{i k}^{1}+c_{m j}^{1}\right) X_{i j k m}^{t}
\end{array}
$$

$$
\begin{aligned}
& X_{i j k m}^{t} \leq y_{k} \quad \forall \mathrm{i}, \mathrm{j}, \mathrm{k}, \mathrm{m} \in \mathrm{N}, \mathrm{t} \in \mathrm{T} \\
& X_{i j k m}^{t} \leq y_{m} \quad \forall \mathrm{i}, \mathrm{j}, \mathrm{k}, \mathrm{m} \in \mathrm{N}, \mathrm{t} \in \mathrm{T} \\
& \sum_{k \in N} y_{k}=p \\
& \sum_{k, m \in N, t \in T} X_{i j k m}^{t}=1-X_{i j}^{1} \quad \forall \mathrm{i}, \mathrm{j} \in \mathrm{N} \\
& X_{i j k m}^{t} \leq S_{k t} \quad \forall \mathrm{i}, \mathrm{j}, \mathrm{k}, \mathrm{m} \in \mathrm{N}, \mathrm{t} \in \mathrm{T} \\
& X_{i j k m}^{t} \leq S_{m t} \quad \forall \mathrm{i}, \mathrm{j}, \mathrm{k}, \mathrm{m} \in \mathrm{N}, \mathrm{t} \in \mathrm{T} \\
& \sum_{k \neq m \in N, t \in T} X_{i j k m}^{t}\left[T_{i k}^{1}+\sum_{O \in R_{O}} W_{k}^{O}(X)+\sum_{o \in R_{O}} S_{k}^{O}+T_{k m}^{t}+\sum_{O \in R_{I}} W_{m}^{O}(X)+\sum_{O \in R_{I}} s_{m}^{O}+T_{m j}^{1}\right]+ \\
& X_{i j}^{1} T_{i j}^{1} \leq T W_{i j} \quad \forall \mathrm{i}, \mathrm{j} \in \mathrm{N} \\
& \sum_{k \in N} X_{i j k k}^{1}\left[T_{i k}^{1}+\sum_{O \in R_{L}} W_{k}^{O}(X)+\sum_{O \in R_{L}} s_{k}^{O}+T_{k j}^{1}\right]+X_{i j}^{1} T_{i j}^{1} \leq T W_{i j} \quad \forall \mathrm{i}, \mathrm{j} \in \mathrm{N} \\
& y_{k}, X_{i j}^{1}, X_{i j k m}^{t}, S_{k t} \in\{0,1\} \quad \forall \mathrm{i}, \mathrm{j}, \mathrm{k}, \mathrm{m} \in \mathrm{N}, \mathrm{t} \in \mathrm{T}
\end{aligned}
$$

Constraint 1 and 2 ensures between spoke $\mathrm{i}$ and spoke $\mathrm{j}$ is hub. Constraint 3 means there are $\mathrm{p}$ hubs in the network. Constraint 4 is to ensure the freight path between the nodes in the network, and there are only one transportation mode. Constraint 5 ensures any value is 1 , the hub $\mathrm{k}$ will provide 1 transportation with mode $\mathrm{t}$, and the variable value $S_{\mathrm{kt}}$ is 1 . Constraint 6 ensures that once goods have arrived at hub m with transport mode t, than the variable value $S_{m t}$ is 1 . Constraint 7 is the total transport time, and constraint 8 is the service time limit. Constraint 9 ensures the decision variables value for 1 or 0 .

3.2. The lower model

The lower model assumes that the following:

(1)The goods in a different hub, with different processes. (2) Goods have reached the hub in the time intervals are independent and identically distributed, the processing time for each service desk is also independent and is subject to General Service time distribution. (3) Different goods in the system in accordance with their respective processes, in turn, and eventually left the hub to the next node of the city. (4) Transport from the origin city to the city of destination can only choose a particular mode of transport. (5) Average service time of the service desks in the system only related to its services and the number of services.

Parameters are defined as follows:

$\mathrm{N}$ — city spoke;

$\mathrm{T}$ — transport mode, $\mathrm{t} \in \mathrm{T}$;

$\mathrm{H}$ — the service desk, $\mathrm{H}=\{1,2,3,4\}, \mathrm{h} \in \mathrm{H}, \mathrm{h}=1,2,3,4$, Representing the unloading, delivery, sorting and loading service desk;

$\mu_{k}^{h}$ — Service rate of the service desk $\mathrm{h}$ in hub k;

$c s_{k}^{h}$ — the square of the time variable coefficient; 
$\lambda_{k}^{h}$ — the arrival rate of the service desk h;

$c a_{k}^{h}$ — the square of the time of arrival variable coefficient;

$c d_{k}^{h}$ — the square of the leave time variable coefficient;

$\lambda_{\mathrm{kmt}}^{O}$ — the arrival rate of hub outbound goods;

$\lambda_{k \mathrm{t}}^{I}$ — the arrival rate of hub inbound goods;

$\lambda_{k}^{L}$ — the arrival rate of hub local goods;

c- - goods transit time;

$s_{k}^{h}$ — the average service time for goods at the service desk h;

$W_{k}^{h}$ — the wait time for goods at the service desk h;

The daily reach rate and average service time of the hub outbound goods, hub inbound goods and hub local goods is calculated as follows:

$$
\begin{aligned}
& \lambda_{k m t}^{O}=\frac{1}{D} \sum_{i, j \in N} f_{i j} X_{i j k m}^{t} \quad \forall \mathrm{k}, \mathrm{m} \in \mathrm{N}(\mathrm{k} \neq \mathrm{m}), \mathrm{t} \in \mathrm{T} \\
& \lambda_{k t}^{I}=\frac{1}{D} \sum_{i, j, m \neq k \in N} f_{i j} X_{i j m k}^{t} \quad \forall \mathrm{k} \in \mathrm{N}, \mathrm{t} \in \mathrm{T} \\
& \lambda_{k}^{L}=\frac{1}{D} \sum_{i, j \in N} f_{i j} X_{i j k k}^{t} \quad \forall \mathrm{k} \in \mathrm{N} \\
& s_{k}^{h}=f_{i j} /\left(D \times \mu_{k}^{h}\right) \quad \forall \mathrm{k} \in \mathrm{N}, \mathrm{h} \in \mathrm{H}
\end{aligned}
$$

$\mathrm{D}$ for the year service days, $f_{i j}$ for the annual freight sending between city $\mathrm{i}$ and city $\mathrm{j}$ of destination.

(1) $\mathrm{h}=1$

$$
\begin{gathered}
\lambda_{k}^{1}=\sum_{m \in N, t \in T} \lambda_{k m t}^{O}+\lambda_{k}^{L}+\sum_{t \in T} \lambda_{k t}^{I} \quad \forall \mathrm{k} \in \mathrm{N} \\
c a_{k}^{1}=\omega_{k}^{1}\left[\sum_{m \in N, t \in T}\left(\frac{\lambda_{k m t}^{O}}{\lambda_{k}^{1}} c\right)+\frac{\lambda_{k}^{L}}{\lambda_{k}^{1}} c+\sum_{t \in T}\left(\frac{\lambda_{k t}^{I}}{\lambda_{k}^{1}} c_{t}\right)\right]+1-\omega_{k}^{1} \\
c d_{k}^{1}=\left(\rho_{k}^{1}\right)^{2} c s_{k}^{1}+\left(1-\left(\rho_{k}^{1}\right)^{2}\right) c a_{k}^{1} \\
W_{k}^{1}=\left(\frac{c a_{k}^{1}+c s_{k}^{1}}{2}\right)\left(\frac{\lambda_{k}^{1}}{\mu_{k}^{1}\left(\mu_{k}^{1}-\lambda_{k}^{1}\right)}\right) g\left(\rho_{k}^{1}, c a_{k}^{1}, c s_{k}^{1}\right) \quad \forall \mathrm{k} \in \mathrm{N} \\
\omega_{k}^{1}=\left[1+4\left(1-\rho_{k}^{1}\right)^{2}\left(v_{k}^{1}-1\right)\right]^{-1}, \\
v_{k}^{1}=\left[\sum_{m \in N, t \in T}\left(\frac{\lambda_{k m t}^{O}}{\lambda_{k}^{1}}\right)^{2}+\left(\frac{\lambda_{k}^{L}}{\lambda_{k}^{1}}\right)^{2}+\sum_{t \in T}\left(\frac{\lambda_{k t}^{I}}{\lambda_{k}^{1}}\right)^{2}\right]^{-1}, \\
\rho_{k}^{1}=\frac{\lambda_{k}^{1}}{\mu_{k}^{1}}, g\left(\rho_{k}^{1}, c a_{k}^{1}, c s_{k}^{1}\right)=\left\{\begin{array}{cl}
\frac{-2\left(1-\rho_{k}^{1}\right)\left(1-c a_{k}^{1}\right)^{2}}{3 \rho_{k}^{1}\left(c a_{k}^{1}+c s_{k}^{1}\right)} & , c a_{k}^{1}<1 \\
1 \quad c a_{k}^{1} \geq 1
\end{array}\right.
\end{gathered}
$$

(2) $h=2$

Total amount of the waiting time: $\frac{1}{\lambda_{k m t}^{O}}\left[\left(L^{t}-1\right)+\left(L^{t}-2\right)+\cdots+1\right]=\frac{\left(L^{t}-1\right) L^{t}}{2 \lambda_{k m t}^{O}}$ 。

(3) $h=3$

$$
\begin{gathered}
W_{k}^{b_{m t}}=\frac{\left(L^{t}-1\right)}{2 \lambda_{k m t}^{O}} \quad \forall \mathrm{k}, \mathrm{m} \in \mathrm{N}, \mathrm{t} \in \mathrm{T} \\
c a_{k}^{b_{m t}}=q_{k}^{1, b_{m t}} c d_{k}^{1}+\left(1-q_{k}^{1, b_{m t}}\right) q_{k}^{1, b_{m t}}+\left(1-q_{k}^{1, b_{m t}}\right)^{2} c \\
q_{k}^{1, b_{m t}}=\frac{\lambda_{k m t}^{O}}{\lambda_{k}^{1}}
\end{gathered}
$$

The wait time is a fixed constant $\varepsilon$.

(4) $h=4$

$$
\begin{gathered}
W_{k}^{3}=\varepsilon \quad \forall \mathrm{k} \in \mathrm{N}, \mathrm{t} \in \mathrm{T} \\
c a_{k}^{B_{t}, 4}=q_{k}^{1, B_{t}} c d_{k}^{1}+\left(1-q_{k}^{1, B_{t}}\right) q_{k}^{1, B_{t}}+\left(1-q_{k}^{1, B_{t}}\right)^{2} c_{t} \\
q_{k}^{1, B_{t}}=\frac{\lambda_{k t}^{I}}{\lambda_{k}^{1}}
\end{gathered}
$$

$$
\lambda_{k}^{4}=\sum_{m \in N, t \in T} \lambda_{k m t}^{O}+\lambda_{k}^{L}+\sum_{t \in T} \lambda_{k t}^{I} \quad \forall \mathrm{k} \in \mathrm{N}
$$




$$
\begin{gathered}
c a_{\mathrm{k}}^{4}=\omega_{k}^{4}\left[\sum_{m \in N, t \in T}\left(\frac{\lambda_{k m t}^{o}}{\lambda_{k}^{4} L^{t}} c a_{k}^{b_{m} t}\right)+\sum_{t \in T}\left(\frac{\lambda_{k t}^{I}+\lambda_{k}^{L}}{\lambda_{k}^{4}} c a_{k}^{B_{t}, 4}\right)\right]+1-\omega_{k}^{4} \\
W_{k}^{4}=\left(\frac{c a_{k}^{4}+c s_{k}^{4}}{2}\right)\left(\frac{\lambda_{k}^{4}}{\mu_{k}^{4}\left(\mu_{k}^{4}-\lambda_{k}^{4}\right)}\right) g\left(\rho_{k}^{4}, c a_{k}^{4}, c s_{k}^{4}\right) \quad \forall \mathrm{k} \in \mathrm{N} \\
\omega_{k}^{4}=\left[1+4\left(1-\rho_{k}^{4}\right)^{2}\left(v_{k}^{4}-1\right)\right]^{-1}, \\
v_{k}^{4}=\left[\sum_{m \in N, t \in T}\left(\frac{\lambda_{k m t}^{O}}{\lambda_{k}^{4}}\right)^{2}+\left(\frac{\lambda_{k}^{L}}{\lambda_{k}^{4}}\right)^{2}+\sum_{t \in T}\left(\frac{\lambda_{k t}^{I}}{\lambda_{k}^{4}}\right)^{2}\right]^{-1}, \\
\rho_{k}^{4}=\frac{\lambda_{k}^{4}}{\mu_{k}^{4}} \quad, g\left(\rho_{k}^{4}, c a_{k}^{4}, c s_{k}^{4}\right)=\left\{\begin{array}{cl}
\frac{-2\left(1-\rho_{k}^{4}\right)\left(1-c a_{k}^{4}\right)^{2}}{3 \rho_{k}^{1}\left(c a_{k}^{4}+c s_{k}^{4}\right)} & , c a_{k}^{4}<1 \\
1 & , c a_{k}^{4} \geq 1
\end{array}\right.
\end{gathered}
$$

Total average waiting time, and the average service time at different desks waiting for the time and services combined.

\section{The tabu search algorithm}

4.1 The process of algorithm

Questions were divided into hub location problem $\mathrm{L}(\mathrm{P})$ and routing distribution problem $\mathrm{A}(\mathrm{H})$. Algorithm design process as shown in Figure 2.

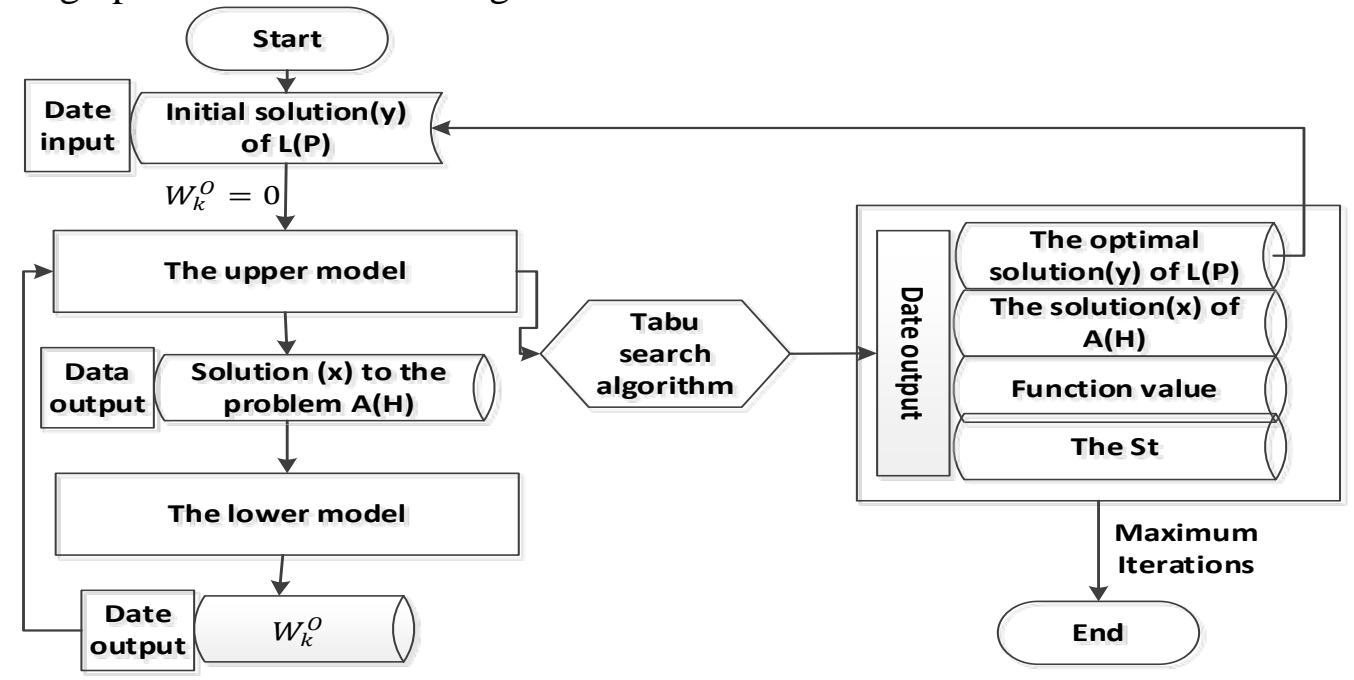

Fig. 2 the design procedure of the algorithm

Tabu search hub location problem in each case $\mathrm{L}(\mathrm{P})$ from the current iteration of the solution to a new solution in the process, we will consider the hub distribution issues $A(H)$. By uninstalling, queuing theory model for delivery wait time formulas, sorting and loading, calculation of goods in the Terminal waiting for time to resolve $\mathrm{A}(\mathrm{H})$ problem . Firstly, assume that the waiting time $W_{k}^{O}=0$, than identifying each pair of cities minimum-cost path by location model and generate an initial solution for distribution problem $\mathrm{A}(\mathrm{H})$. Can be calculated by formula in queuing theory model of the goods in the hub of the wait time and wait time in the location problem $\mathrm{L}(\mathrm{P})$, for the next iteration. This repeated iteration, until it reaches the maximum number of iterations, tabu search terminates.

\subsection{Solution scheme}

Location problem $\mathrm{L}(\mathrm{P})$ solution with $\mathrm{Nx} 3(0,1)$ matrices to represent, where the left column represents the radiation nodes, the middle column represents the rode hub, right column representative of rail intermodal hubs and a city can only establish a hub, that at most one element in each row is 1 . Establish a cell array $\mathrm{X}$ to represent the allocation of path problem solution. Solving process using hub between nodes and radiation into the Exchange as a neighborhood search method. Solution evaluation functions entrusted to tabu search each solution in the space of a real-valued, which values $(Z)$, the pros and cons of the solution is by comparing the values determined by the size. TabuLength, the $\mathrm{L}=\sqrt{P *(\text { CityNum }-\mathrm{P})}$ rounded to integer. If all 
candidate solutions is banned and there are no better than the candidate solutions of the optimal solution, using Amnesty guidelines to ban candidates, and as the current solution, in order to continue the search. And maximum number of iterations as the standard algorithm stops.

\section{Model algorithm test}

6 cities number of Arabic numerals 1 to 6 respectively, choose 2 from the 6 cities to hub city. Specific data is as follows:

\begin{tabular}{c|cccccc}
\multicolumn{8}{c}{ Table 1 The coordinates of the city nodes } \\
\hline & 1 & 2 & 3 & 4 & 5 & 6 \\
\hline $\mathrm{X}$ & 60 & 110 & 10 & 48 & 72 & 60 \\
$\mathrm{Y}$ & 97 & 10 & 10 & 50 & 50 & 20 \\
\hline
\end{tabular}

\begin{tabular}{c|cccccc}
\hline \multicolumn{7}{c}{ Table 2 The freight volume } \\
\hline City & 1 & 2 & 3 & 4 & 5 & 6 \\
\hline 1 & 0 & 10 & 16 & 114 & 166 & 176 \\
2 & 10 & 0 & 97 & 142 & 104 & 174 \\
3 & 16 & 97 & 0 & 192 & 185 & 139 \\
4 & 114 & 142 & 192 & 0 & 193 & 166 \\
5 & 166 & 104 & 185 & 193 & 0 & 117 \\
6 & 176 & 174 & 139 & 166 & 117 & 0 \\
\hline
\end{tabular}

\begin{tabular}{c|cccccc}
\hline \multicolumn{7}{c}{ Table 3 The road freight among cities } \\
\hline City & 1 & 2 & 3 & 4 & 5 & 6 \\
\hline 1 & 0 & 398 & 249 & 35 & 24 & 23 \\
2 & 398 & 0 & 41 & 28 & 38 & 23 \\
3 & 249 & 41 & 0 & 21 & 22 & 29 \\
4 & 35 & 28 & 21 & 0 & 21 & 24 \\
5 & 24 & 38 & 22 & 21 & 0 & 34 \\
6 & 23 & 23 & 29 & 24 & 34 & 0 \\
\hline
\end{tabular}

\begin{tabular}{c|cccccc}
\hline \multicolumn{7}{|c}{ Table 4 The rail freight } \\
\hline City & 1 & 2 & 3 & 4 & 5 & 6 \\
\hline 1 & 0 & 597 & 374 & 53 & 12 & 11 \\
2 & 597 & 0 & 62 & 14 & 57 & 11 \\
3 & 374 & 62 & 0 & 10 & 11 & 14 \\
4 & 53 & 14 & 10 & 0 & 10 & 12 \\
5 & 12 & 57 & 11 & 10 & 0 & 51 \\
6 & 11 & 11 & 14 & 12 & 51 & 0 \\
\hline
\end{tabular}

Table 5 The road transportation of time

\begin{tabular}{c|cccccc}
\hline City & 1 & 2 & 3 & 4 & 5 & 6 \\
\hline 1 & 0 & 1.25 & 1.25 & 0.6125 & 0.6125 & 0.9625 \\
2 & 1.25 & 0 & 1.25 & 0.925 & 0.6875 & 0.6375 \\
3 & 1.25 & 1.25 & 0 & 0.6875 & 0.925 & 0.6375 \\
4 & 0.6125 & 0.925 & 0.6875 & 0 & 0.3 & 0.4 \\
5 & 0.6125 & 0.6875 & 0.925 & 0.3 & 0 & 0.4 \\
6 & 0.9625 & 0.6375 & 0.6375 & 0.4 & 0.4 & 0 \\
\hline \multicolumn{7}{c}{ Table 6 The rail transportation of time } \\
\hline City & 1 & 2 & 3 & 4 & 5 & 6 \\
\hline 1 & 0 & 1 & 1 & 0.49 & 0.49 & 0.77 \\
2 & 1 & 0 & 1 & 0.74 & 0.55 & 0.51 \\
3 & 1 & 1 & 0 & 0.55 & 0.74 & 0.51 \\
4 & 0.49 & 0.74 & 0.55 & 0 & 0.24 & 0.32 \\
5 & 0.49 & 0.55 & 0.74 & 0.24 & 0 & 0.32 \\
6 & 0.77 & 0.51 & 0.51 & 0.32 & 0.32 & 0 \\
\hline
\end{tabular}


Table 7 The limitation of the service time

\begin{tabular}{|c|c|c|c|c|c|c|c|}
\hline City & 1 & 2 & \multicolumn{2}{|c|}{3} & 4 & 5 & 6 \\
\hline 1 & 0 & 5 & \multicolumn{2}{|c|}{5} & 2.45 & 2.45 & 3.85 \\
\hline 2 & 5 & 0 & \multicolumn{2}{|c|}{5} & 3.7 & 2.75 & 2.55 \\
\hline 3 & 5 & 5 & \multicolumn{2}{|c|}{0} & 2.75 & 3.7 & 2.55 \\
\hline 4 & 2.45 & 3.7 & \multicolumn{2}{|c|}{2.75} & 0 & 1.2 & 1.6 \\
\hline 5 & 2.45 & 2.75 & \multicolumn{2}{|c|}{3.7} & 1.2 & 0 & 1.6 \\
\hline 6 & 3.85 & 2.55 & \multicolumn{2}{|c|}{2.55} & 1.6 & 1.6 & 0 \\
\hline \multicolumn{8}{|c|}{ Table 8 The fixed cost parameter in the model } \\
\hline & & 1 & 2 & 3 & 4 & 5 & 6 \\
\hline \multicolumn{2}{|c|}{$F_{k}$} & 750 & 770 & 740 & 720 & 710 & 700 \\
\hline \multicolumn{2}{|c|}{ Rode $M C_{k 1}$} & 150 & 130 & 140 & 120 & 100 & 110 \\
\hline \multicolumn{2}{|c|}{ Railway $M C_{k 2}$} & 350 & 340 & 330 & 310 & 300 & 320 \\
\hline
\end{tabular}

Design length of tabu search algorithm Tabu Length $=\sqrt{2 *(6-2)} \approx 3$, candidate solutions focused on the number of solutions for 8 , maximum number of iterations is 48 , by using the MATLAB computing, convergence objective function curve is shown in Figure 3, the objective function and the values for the decision variables as shown in Figure 4.

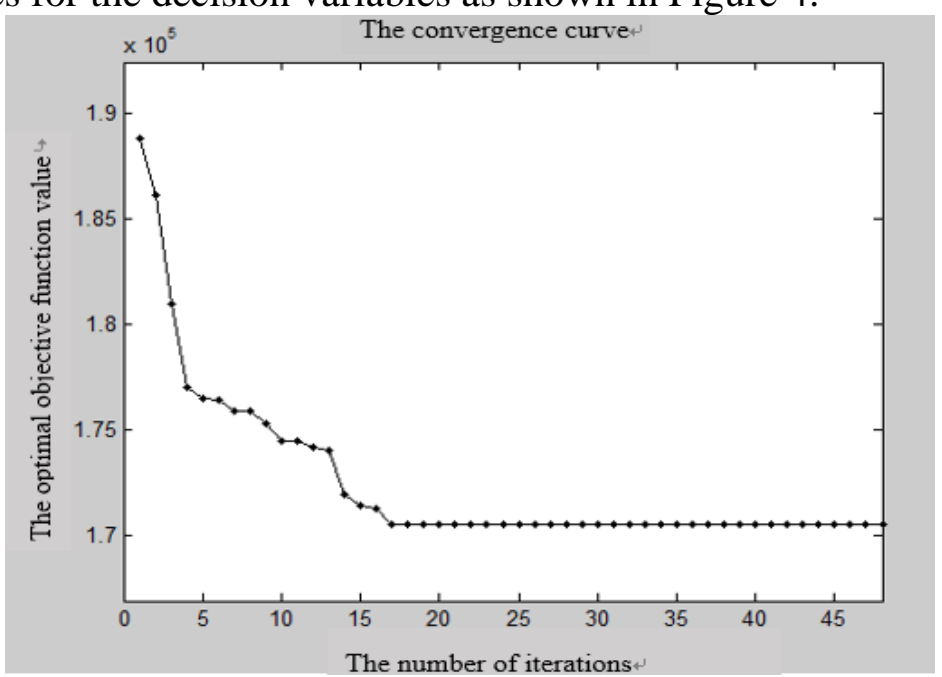

Fig. 3 the optimal objective function convergence curve

$\begin{array}{ll}\text { Name } & \text { Value } \\ \boxplus \text { MinZ } & 48 \times 1 \text { double } \\ \boxplus \text { minsolution } & 170570 \\ \boxplus \text { st } & {[0,0,0,0,0,0]} \\ \square \text { x } & 6 x 6 \mathrm{ce} / / \\ \boxplus \mathrm{y} & {[0,0,0,0,1,1]}\end{array}$

Fig. 4 the value of the objective function and decision variables

Optimal target function value in Diego generation 15 times around convergence, Min Z matrix in the store of is each Diego generation by corresponds to of optimal target function value, min solution=170570 for eventually solution out of optimal target function value, $y$ for hub selected of decision variable, by corresponds to of hub city select for city 5 and 6 , this also and we prior assumes that of results phase consistent, validation has this papers model and algorithm of effectiveness. According to the cell matrix $\mathrm{x}$, algorithm designing policy expressions, rendering nodes in some cities of the optimal route of goods distribution. 


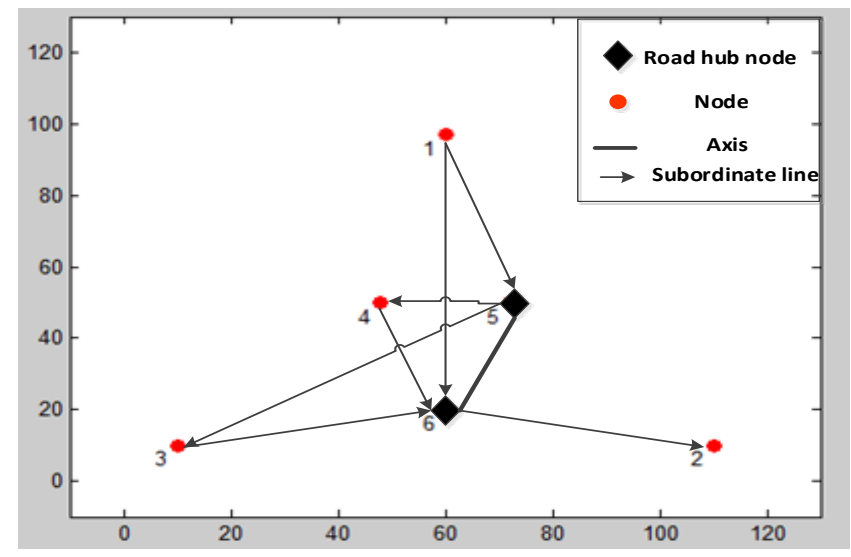

Fig. 5 the partial optimal routing distribution mode among cities

The results show: radiation transport through a hub between nodes for transit traffic. Goods routes from city 1 to the city 2 for 1-6-2; city 1 to city 3 for 1-5-3; city 4 to city 2 for 4-6-2; city 1 to 4 for 1-5-4.

\section{Summary}

Paper consider three species path distribution way (direct transport, and single hub transport and double Hub Transport), two species different transport way (Road Transport and railway transport), two species different of hub type (Road hub and "road-railway" transport hub), and hub fixed cost, and transport cost, and scale effect, and goods in hub within of queued waiting for time and service time factors,, building axis spoke type logistics network hub location and the path distribution of double planning model, and on this model for heuristic solution algorithm design and test.

In this paper, there are still some areas in need of further refinement and research, mainly reflected in the following aspects: A single type of goods; Network in this model total cost does not take into account the goods arrive to the hub when waiting for the inventory cost, etc.

\section{References}

[1] O’ Kelly, M.E. A quadratic integer program for the location of interacting hub facilities [J]. European Journal of Operational Research 1986 (3) : 393-404.

[2] Campbell, James F. Integer Programming Formulations of Discrete Hub Location Problems [J], European Journal of Operational Research, 1996 (72) : 387-405.

[3] Julia, R., Carsten, E, Jurgen, Z, Many-to-many location-routing with inter-hub transport and multi-commodity pickup-and-delivery [J]. European Journal of Operational Research, 2014(236) : 863-878. 